\title{
Associations between Overall and Abdominal Obesity and Suicidal Ideation among US Adult Women
}

\author{
Guixiang Zhao, ${ }^{1}$ Chaoyang Li, ${ }^{1}$ Earl S. Ford, ${ }^{2}$ James Tsai, ${ }^{2}$ Satvinder S. Dhingra, \\ Janet B. Croft, ${ }^{2}$ Lela R. McKnight-Eily, ${ }^{2}$ and Lina S. Balluz ${ }^{1}$
}

\author{
${ }^{1}$ Division of Behavioral Surveillance, Public Health Surveillance Program Office, Office of Surveillance, \\ Epidemiology and Laboratory Services, Centers for Disease Control and Prevention, Atlanta, GA 30333, USA \\ ${ }^{2}$ Division of Population Health, National Center for Chronic Disease Prevention and Health Promotion, \\ Centers for Disease Control and Prevention, Atlanta, GA 30333, USA
}

Correspondence should be addressed to Guixiang Zhao, fwj4@cdc.gov

Received 19 December 2011; Revised 23 February 2012; Accepted 5 March 2012

Academic Editor: Austin S. Baldwin

Copyright ( 92012 Guixiang Zhao et al. This is an open access article distributed under the Creative Commons Attribution License, which permits unrestricted use, distribution, and reproduction in any medium, provided the original work is properly cited.

\begin{abstract}
Obesity is associated with increased risks for mental disorders. This study examined associations of obesity indicators including body mass index (BMI), waist circumference, and waist-height ratio with suicidal ideation among U.S. women. We analyzed data from 3,732 nonpregnant women aged $\geq 20$ years who participated in the 2005-2008 National Health and Nutrition Examination Survey. We used anthropometric measures of weight, height, and waist circumference to calculate BMI and waist-height ratio. Suicidal ideation was assessed using the Item 9 of the Patient Health Questionnaire-9. Odds ratios with $95 \%$ conference intervals were estimated using logistic regression analyses after controlling for potential confounders. The age-adjusted prevalence of suicidal ideation was 3.0\%; the prevalence increased linearly across quartiles of BMI, waist circumference, and waist-height ratio ( $P$ for linear trend $<0.01$ for all). The positive associations of waist circumference and waist-height ratio with suicidal ideation remained significant $(P<0.05)$ after adjustment for sociodemographics, lifestyle-related behavioral factors, and having either chronic conditions or current depression. However, these associations were attenuated after both chronic conditions and depression were entered into the models. Thus, the previously reported association between obesity and suicidal ideation appears to be confounded by coexistence of chronic conditions and current depression among women of the United States.
\end{abstract}

\section{Introduction}

The rising tide of obesity continues to be a major concern of public health in the USA and worldwide. The prevalence of obesity, defined usually by a body mass index (BMI) of $\geq 30 \mathrm{~kg} / \mathrm{m}^{2}$, has increased substantially from $13.3 \%$ during 1960-1962 to $33.8 \%$ during $2007-2008$ among US adults [1-3] with noticeable differences between men and women (32\% versus $36 \%$ in 2007-2008) [1]. In addition to its substantial impact on physical health (i.e., obesity-related chronic conditions), obesity is also associated with increased risks of psychiatric or mental disorders such as depression, anxiety, mania, panic attacks, and social phobia in both men and women [4-8].

With regard to suicidal behaviors and suicide mortality, a vast majority of previous studies including large, prospective cohort studies have shown that among men, BMI is inversely associated with suicidal ideation, suicide attempt, or suicide death and thus provides protection from suicide mortality [4, 9-13]. Although the mechanism underlying the inverse relationship remains unknown, evidence suggests that low levels of blood cholesterol and markers of insulin sensitivity in lean men may interfere with circulating tryptophan metabolism and brain serotonin production, thereby increasing the risk for suicide attempts and suicide death [14-17]. Among women, a longitudinal follow-up study using the National Health Interview Survey Linked Mortality data reported that for a $5 \mathrm{~kg} / \mathrm{m}^{2}$ increase in BMI, the relative risk of suicide death decreased by $24 \%$ after controlling for sociodemographics, region of residence, number of chronic conditions, number of psychiatric conditions, activity limitation, and self-rated health [10]. However, the National Longitudinal 
Alcohol Epidemiologic Survey study showed that a $10 \mathrm{~kg} / \mathrm{m}^{2}$ increase in BMI was associated with $22 \%$ increased likelihood for past-year suicidal ideation among adult US women after controlling for sociodemographics, lifetime disease history, and substance use [18]. Another study conducted on Canadian women also reported that obesity was associated with increased likelihoods of lifetime and past-year suicidal ideation and suicide attempts when controlling for age, education, psychiatric disorders and comorbidity, and physical illness burden assessed as Charlson Comorbidity Index [6]. Thus, the associations between BMI and suicidal behaviours or suicide death among women remain uncertain. Moreover, in the previous studies, only BMI as an indicator of overall obesity was evaluated; the association between abdominal obesity and suicidal behaviors is largely unknown. To further shed light on these issues, the present study, by using a large nationally representative sample, aimed to (1) examine the prevalence of suicidal ideation among US adult women across quartiles of BMI, a measure of overall obesity, and across quartiles of waist circumference and waist-height ratio (WHR), measures of abdominal obesity, and (2) examine the associations of BMI, waist circumference, and WHR with suicidal ideation while controlling for multiple potential confounders including sociodemographic characteristics, lifestyle-related behavioral risk factors, obesity-related chronic physical conditions, and current depression status, most of which have been included as covariates in the previous studies $[6,10,18]$. We hypothesized that a positive association may exist between abdominal obesity and suicidal ideation as well as between overall obesity and suicidal ideation independent of behavioral risk factors, obesityrelated chronic physical conditions, and current depression status among US women.

\section{Subjects and Methods}

We analyzed data from the National Health and Nutrition Examination Survey (NHANES) 2005-2008, a nationally representative sample obtained using a multistage stratified sampling design from the noninstitutionalized civilian US population. Survey participants were initially interviewed at home and were then invited to a mobile examination center, where they received various examinations and provided blood samples for laboratory tests. Details about the NHANES survey design and operation have been described elsewhere [19]. All procedures involving human subjects were reviewed and approved by the Research Ethics Review Board of the National Center for Health Statistics at the Centers for Disease Control and Prevention. Written informed consent was obtained from all participants.

We examined interview data from women aged $\geq 20$ years who attended the mobile examination center. Anthropometric measurements were performed by trained health technicians. Weight was measured in pounds on a Toledo digital scale with participants only wearing underwear, disposable paper gowns, and foam slippers. Standing height was measured with a stadiometer to the nearest $0.1 \mathrm{~cm}$. BMI was calculated from measured weight $(\mathrm{kg})$ and height $(\mathrm{m})$. Waist circumference was measured at a point immediately above the iliac crest on the midaxillary line at minimal respiration to the nearest $0.1 \mathrm{~cm}$. Waist-height ratio (WHR) was calculated from measured waist circumference $(\mathrm{cm})$ and height $(\mathrm{cm})$. The quartiles of BMI, waist circumference, and WHR were created based on their distributions among women aged $\geq 20$ years after taking into account the sampling weights.

Suicidal ideation was assessed using the 9th item of the Patient Health Questionnaire-9 (PHQ-9) [20, 21], which has been used widely to assess suicidal ideation in psychiatric research [22-26]. Specifically, participants were asked about how often over the previous two weeks they had been bothered by thoughts of being better off dead or of hurting themself in some way. Their response options were categorized as (1) not at all, (2) several days, (3) more than half the days, and (4) nearly every day. Participants with an affirmative response to the options (2) to (4) were defined as having suicidal ideation.

Sociodemographic variables in the analyses included age, sex, race/ethnicity (non-Hispanic white, non-Hispanic black, Mexican American, and other race), educational status ( $<$ high school diploma, high school graduate, and $>$ high school diploma), marital status (married or living with a partner, divorced/widowed/separated, and never married), and family poverty-income ratio (calculated as a ratio of family income to poverty threshold and categorized as $<1.0,1.0-<3.0, \geq 3.0$ ). Health-related behavioral risk factors included smoking, physical activity, and alcohol use, which were assessed based on participants' self-reports. For smoking, participants were asked if they had smoked at least 100 cigarettes in their entire life and if they were smoking cigarettes now. Participants were then categorized as current smokers (those who had smoked at least 100 cigarettes during their lifetime and were still smoking), former smokers (those who had smoked at least 100 cigarettes during their lifetime but stopped), and never smoked (those who had smoked less than 100 cigarettes during their lifetime). Physical activity was assessed by asking participants whether, over the past 30 days, (1) they had engaged in specific moderate or vigorous leisure-time activities, (2) they had walked or bicycled as part of getting to and from work, or school, or to do errands (transportation activities), and (3) they had done any tasks in or around their home or yard for at least 10 minutes that required moderate or greater physical effort (household activities). If a confirmative answer of "yes" was recorded, participants were then asked about how many times and the average duration each time they engaged in the activities over the past 30 days. Based on the Metabolic equivalent task (MET) score for specific activities, we calculated the average daily metabolic equivalent-hour index (MET-hr/day) that summed transportation, household, and leisure-time physical activity and participants were then dichotomized as physically active (MET-hr/day >0) and inactive $($ MET-hr/day $=0)$. Alcohol consumption was assessed by asking respondents how many days per week or per month they had had at least 1 drink (equivalent to a 12-ounce beer, a 5-ounce glass of wine, or a drink with 1 shot of liquor) of any alcoholic beverages during the past 30 days and how many drinks they had on average on the days when they drank. We calculated the average number of daily 
drinks, and participants were then dichotomized as having excessive drinking ( $>1$ drink/day) and not ( $\leq 1$ drink/day).

Chronic conditions included hypertension, diabetes, coronary artery disease (i.e., coronary heart disease, angina pectoris, or heart attack), congestive heart failure, stroke, arthritis, asthma, chronic bronchitis, emphysema, thyroid problem, liver disease, renal disease or renal failure, sleep disorders, disability, and cancer. Most of the conditions were assessed by asking participants whether they had ever been told by a healthcare professional that they had these conditions or whether they still had asthma, chronic bronchitis, thyroid problem, or liver disease at the time when the survey was conducted. Mean systolic and diastolic blood pressures were calculated as the average of the last two readings of systolic or diastolic blood pressure for participants who had three measurements, as the last reading for participants who had two measurements, and as the only reading for participants who had one measurement. Participants who were on antihypertension medications or had systolic blood pressure of $\geq 140 \mathrm{mmHg}$ or diastolic blood pressure of $\geq 90 \mathrm{mmHg}$ were defined as having hypertension [27]. Disability status was assessed by asking participants whether (1) they were limited in any way in any activities including limitations from working and walking or experiencing confusion/memory problems because of physical, mental, or emotional problems and (2) they were required to use special equipments such as a cane, a wheelchair, a special bed, or a special telephone because of any health problem. Participants with an affirmative response to either question were defined as having a disability. The number of the above chronic conditions was summed and participants were defined as having none of the conditions, 1-2 conditions, or $\geq 3$ conditions.

Current depressive symptoms were assessed using the items 1 to 8 of the PHQ-9, which has been shown to provide a valid measure of depressive symptoms and severity in the general population [28]. Briefly, participants were asked about symptoms of depression they experienced in the past 2 weeks, which included little interest or pleasure in doing things; feeling down, depressed, or hopeless; trouble falling asleep, staying asleep, or sleeping too much; feeling tired or having little energy; a poor appetite or eaten too much; feeling bad as a failure or letting self or family down; trouble concentrating on things such as reading the newspaper or watching TV; and moving or speaking too slowly, or moving around a lot more than usual because of being so fidgety or restless. A total depression score was calculated as described elsewhere [7, 28, 29]; participants with a score of $\geq 10$ were defined as having current depression. The scoring of $\geq 10$ has a sensitivity and specificity of $88 \%$ for major depression [21].

\section{Statistical Analysis}

The prevalence of suicidal ideation was estimated and agestandardized to the 2000 female population in the USA The regression coefficient $(\beta)$, odds ratio (OR), and $95 \%$ confidence interval (CI) for having suicidal ideation were estimated by conducting logistic regressions using each obesity indicator (BMI, waist circumference, and WHR) as a predictor while controlling for covariates including sociodemographic characteristics, lifestyle-related behaviors, chronic conditions, and current depression. A body of evidence has shown that obesity is associated with a variety of risky lifestyle behaviors (such as physical inactivity and alcohol use) and health outcomes (such as obesity-related chronic physical conditions and mental disorders) $[7,8,30$ 37]. On the other hand, the sociodemographic factors, risky lifestyle behaviors, stressful life events including severe chronic or terminal illness, and psychiatric and psychological factors are important risk factors related to suicidal ideation or suicidal behaviors [38-41]. Therefore, we included these variables as study covariates in our analyses; similar covariates have also been used in the previous studies.

Trends in the prevalence of suicidal ideation were tested using orthogonal contrasts, and the trends in the ORs were tested using the median values for the quartiles of BMI, waist circumference and WHR in logistic regression models. SUDAAN (Software for the Statistical Analysis of Correlated Data, Release 9.0, Research Triangle Institute, Research Triangle Park, NC) was used to account for the complex sampling design.

\section{Results}

Among 5,399 female participants aged $\geq 20$ years, exclusions included pregnant women $(n=382)$ and women with missing values for suicidal ideation $(n=537)$, BMI $(n=94)$, waist circumference $(n=307)$, or WHR $(n=310)$. After further excluding those who had missing values for study covariates, 3,732 nonpregnant women (median age: 46 years) remained in our analyses. Approximately $73.7 \%$ were nonHispanic white, $10.8 \%$ non-Hispanic black, $6.7 \%$ Hispanic, and $8.8 \%$ other racial/ethnic participants. About 59.3\% attained an educational level of greater than a high school diploma, $61.8 \%$ were married or living with partners, and $52.2 \%$ had a poverty-income ratio of $\geq 3.0$. The percentages of women with unhealthy lifestyle behaviors were $20.6 \%$ for current smoking, $19.5 \%$ for physical inactivity, and $6.8 \%$ for excessive alcohol drinking. In addition, $39.8 \%$ reported having 1-2 chronic conditions, $23.1 \%$ reported having $\geq 3$ of chronic conditions, and $8.2 \%$ reported having current depression.

Overall, the unadjusted and age-adjusted prevalence of suicidal ideation was 3.1\% (95\% CI: $2.5-3.8 \%$ ) and 3.0\% (95\% CI: $2.4-3.8 \%$ ), respectively (Table 1 ). The prevalence of suicidal ideation was the lowest in non-Hispanic white women or in women who had an educational level of $>$ high school diploma, who were married or living with partners, or who had a poverty-income ratio of $\geq 3.0$ among their respective categories (Bonferroni corrected $P<0.05$ for all). However, the prevalence was significantly higher in women with poor health behaviors $(P<0.05$ for all), chronic conditions $(P<0.01)$, or current depression $(P<0.01)$ compared to their counterparts (Table 1).

The means of the obesity indicators were $28.5 \mathrm{~kg} / \mathrm{m}^{2}$ for BMI, $94.4 \mathrm{~cm}$ for waist circumference, and 0.58 for WHR among study participants. The age-adjusted prevalence of suicidal ideation increased linearly with increasing quartiles of BMI, waist circumference, and WHR ( $P$ for linear 


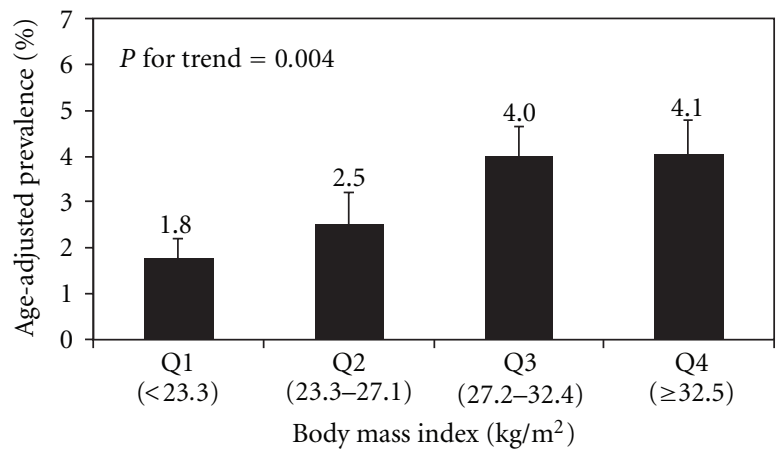

(a)

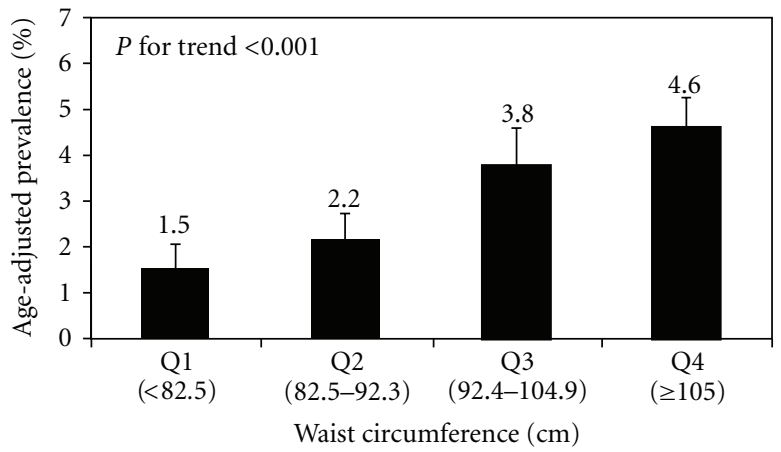

(b)

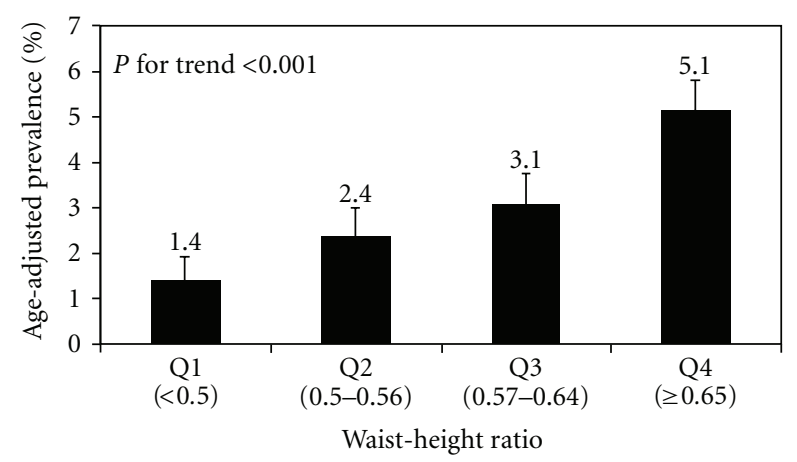

(c)

FIGURE 1: Age-adjusted prevalence (with standard error) of having suicidal ideation among US adult women by quartiles of body mass index, waist circumference, and waist-height ratio, NHANES 2005-2008.

trend $<0.01$ for all) (Figure 1). Similarly, the unadjusted ORs for suicidal ideation also increased linearly across quartiles of BMI, waist circumference, and WHR $(P<0.01$ for all, Model 1, Table 2). Adjustment for sociodemographic variables and lifestyle-related behavioral factors had little effects on these ORs (Model 2). After further adjustment for either chronic conditions (Model 3) or current depression (Model 4), the positive associations of waist circumference and WHR with suicidal ideation were attenuated but remained statistically significant $(P<0.05)$. However, when adjusting simultaneously for both chronic conditions and current depression (Model 5), the strength of the associations was reduced substantially. The association of BMI with suicidal ideation was attenuated after further adjustment for either chronic conditions or current depression or both (Models 3-5). These similar patterns persisted when continuous BMI, waist circumference, and WHR were entered in the models (Table 3 ).

\section{Discussion}

To our knowledge, our study is the first to examine the associations between anthropometric indicators for obesity and suicidal ideation among women in a large, populationbased, nationally representative sample. Although our study demonstrated a significantly increasing trend in the prevalence of suicidal ideation with increasing levels of the three indicators for obesity, the associations between obesity and suicidal ideation seem to be confounded by having chronic conditions or current depression, either individually (for the association between BMI and suicidal ideation) or jointly (for the associations between waist circumference and WHR and suicidal ideation).

Suicide is one of the leading mental health problems in the world with enormous consequence [42, 43]. From 19901992 to 2001-2003, the prevalence of suicidal ideation, plans, or attempts did not vary much in the US population despite a dramatic increase in treatment [44]. In 2007, suicide accounted for more than 34,000 deaths and was the 11th leading cause of death in the United States [42]. Regarding suicidal ideation, results of previous studies showed that the lifetime prevalence of suicidal ideation was $15.6 \%$ in the adult US population [40]. A recent study showed that about $3.7 \%$ of US adults (3.5\% of men and $3.9 \%$ of women) reported having suicidal thoughts in the past year [45]. Our study further revealed that about $3.0 \%$ of US women reported having suicidal ideation in the past 2 weeks.

Multiple risk factors for suicidal ideation or suicidal behaviors have been reported including sociodemographic factors (such as younger age, being female, less educated, and not being married), DSM-IV disorders (such as anxiety disorders, mood disorders, impulse-control disorders, depression, and substance abuse), chronic physical conditions (such as disability, chronic physical pain, terminal illness, and life-threatening diseases), some medications, and firearm ownership [24, 26, 39-41, 46-48]. Previous studies exploring the relationships between BMI and risk of suicide mortality 
TABLe 1: Prevalence estimates of suicidal ideation among US women aged $\geq 20$ years by selected characteristics, NHANES $2005-2008$.

\begin{tabular}{|c|c|c|c|}
\hline & $n$ & $\% *$ & $95 \% \mathrm{CI}$ \\
\hline \multicolumn{4}{|l|}{ Total } \\
\hline Unadjusted & 3,732 & 3.1 & $2.5-3.8$ \\
\hline Age-adjusted & 3,732 & 3.0 & $2.4-3.8$ \\
\hline \multicolumn{4}{|l|}{ Demographic characteristic } \\
\hline \multicolumn{4}{|l|}{ Age (yr) } \\
\hline $20-39$ & 1,195 & 2.5 & $1.6-3.8$ \\
\hline $40-59$ & 1,283 & 3.8 & $2.8-5.3$ \\
\hline$\geq 60$ & 1,254 & 2.6 & $1.7-4.1$ \\
\hline \multicolumn{4}{|l|}{ Race/ethnicity } \\
\hline Non-Hispanic white & 1,866 & $2.2^{\mathrm{a}}$ & $1.4-3.4$ \\
\hline Non-Hispanic black & 787 & $3.7^{\mathrm{ab}}$ & $2.3-5.8$ \\
\hline Mexican American & 664 & $5.8^{\mathrm{b}}$ & $3.7-8.9$ \\
\hline Other & 415 & $6.1^{\mathrm{b}}$ & $3.8-9.6$ \\
\hline \multicolumn{4}{|l|}{ Education } \\
\hline$<$ high school diploma & 971 & $6.5^{\mathrm{a}}$ & $4.7-9.1$ \\
\hline High school graduate & 893 & $3.3^{\mathrm{ab}}$ & $2.0-5.5$ \\
\hline >high school diploma & 1,868 & $2.0^{\mathrm{b}}$ & $1.3-3.0$ \\
\hline \multicolumn{4}{|l|}{ Marital status } \\
\hline Married/living with partner & 2,072 & $2.3^{\mathrm{a}}$ & $1.6-3.1$ \\
\hline Divorced/widowed/separated & 1,115 & $5.0^{\mathrm{b}}$ & $3.3-7.6$ \\
\hline Never married & 545 & $4.1^{\mathrm{ab}}$ & $2.6-6.6$ \\
\hline \multicolumn{4}{|l|}{ Poverty-income ratio } \\
\hline$<1.0$ & 706 & $7.6^{\mathrm{a}}$ & $5.6-10.4$ \\
\hline $1.0-2.9$ & 1,572 & $4.7^{\mathrm{a}}$ & $3.6-6.0$ \\
\hline$\geq 3.0$ & 1,454 & $1.0^{\mathrm{b}}$ & $0.6-1.7$ \\
\hline \multicolumn{4}{|l|}{ Lifestyle-related behavioral factor } \\
\hline \multicolumn{4}{|l|}{ Smoking } \\
\hline Current smoking & 729 & $5.5^{\mathrm{a}}$ & $4.0-7.5$ \\
\hline Former smoking & 736 & $1.4^{\mathrm{b}}$ & $0.8-2.4$ \\
\hline Never & 2267 & $2.6^{\mathrm{b}}$ & $2.0-3.4$ \\
\hline \multicolumn{4}{|l|}{ Excessive drinking } \\
\hline Yes & 206 & $5.9^{\mathrm{a}}$ & $3.8-8.9$ \\
\hline No & 3,526 & $2.8^{\mathrm{b}}$ & $2.2-3.4$ \\
\hline \multicolumn{4}{|l|}{ Physical activity } \\
\hline Yes & 2,758 & $2.6^{\mathrm{a}}$ & $2.0-3.4$ \\
\hline No & 974 & $5.0^{\mathrm{b}}$ & $3.6-6.9$ \\
\hline \multicolumn{4}{|l|}{ Comorbidity } \\
\hline \multicolumn{4}{|l|}{ Number of chronic conditions } \\
\hline 0 & 1,259 & $1.0^{\mathrm{a}}$ & $0.6-1.6$ \\
\hline $1-2$ & 1,457 & $2.7^{\mathrm{b}}$ & $2.0-3.6$ \\
\hline$\geq 3$ & 1,016 & $8.8^{\mathrm{c}}$ & $5.7-13.2$ \\
\hline \multicolumn{4}{|l|}{ Current depression } \\
\hline Yes & 357 & $20.3^{\mathrm{a}}$ & $15.8-25.7$ \\
\hline No & 3,375 & $1.4^{\mathrm{b}}$ & $1.0-2.1$ \\
\hline
\end{tabular}


TABLE 2: Odds ratios with 95\% confidence intervals for suicidal ideation among US women aged $\geq 20$ years by quartiles of BMI, waist circumference, and waist-height ratio, NHANES 2005-2008 $(n=3,732)$.

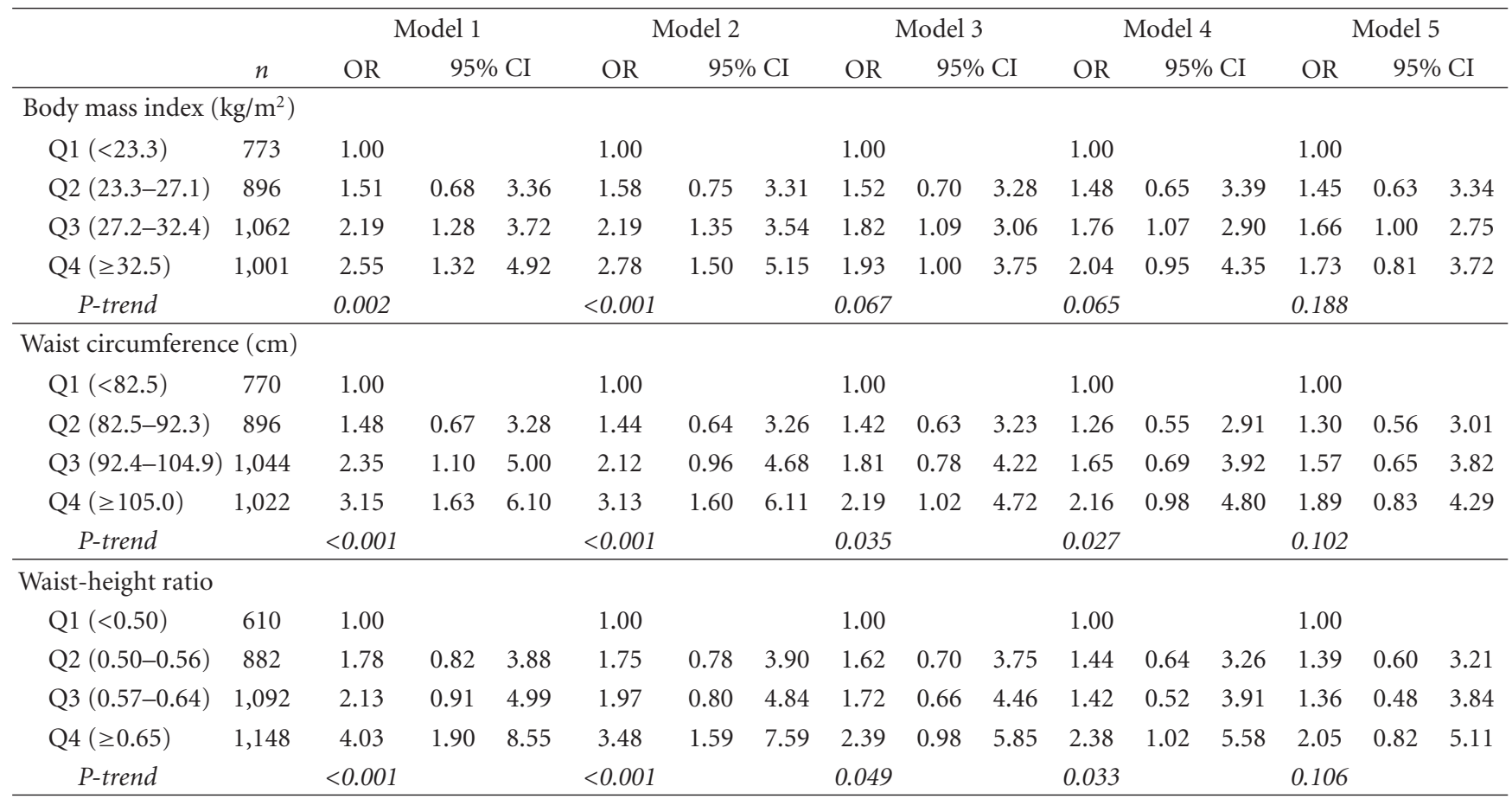

* Model 1: unadjusted; Model 2, adjusted for demographic characteristics (age, race, education, marital status, poverty-income ratio) and lifestyle-related behaviors (current smoking, excessive drinking, and physical activity); Model 3: further adjusted for the number of chronic conditions (including diabetes, hypertension, congestive heart failure, coronary artery disease, stroke, arthritis, asthma, chronic bronchitis, emphysema, liver disease, thyroid disease, renal diseases, sleep disorders, cancer, and disability); Model 4: further adjusted for current depression; Model 5: further adjusted for both the number of chronic conditions and current depression.

or suicidal behaviors among women have yielded mixed results $[6,10,18]$. This may have resulted from different study settings with different outcomes assessed (the risk of suicide death versus lifetime or past-year suicidal ideation or suicidal attempts) and different covariates adjusted for. The present study provides further evidence that a high BMI level was associated with an increased likelihood for suicidal ideation among US adult women independent of sociodemographic variables and lifestyle risk factors; however, this positive association no longer existed after taking into consideration the chronic conditions or current depression status as demonstrated in the present study. Importantly, our results also showed that both chronic conditions and current depression were significantly associated with suicidal ideation. Thus, the previously reported association between BMI and suicidal ideation may have been confounded by existence of obesity-related chronic conditions or depression, which deserves further investigation.

The strength of the present study is that we were able to simultaneously assess the relationships between abdominal obesity as measured by waist circumference and WHR and suicidal ideation. Abdominal obesity has been shown to be associated with impaired health, impaired quality of life, and psychiatric disorders $[5,8,49,50]$. However, results of a previous study showed that waist-hip ratio was not a predictor of suicide mortality among 46,755 male participants in the Health Professionals Follow-up Study [12].
Results of the present study demonstrated that both waist circumference and waist-height ratio were positively associated with suicidal ideation among US adult women irrespective of having either chronic conditions or current depression; however, as shown for BMI, these associations appeared to be confounded jointly by chronic conditions and current deperssion.

Studies have shown that both physical conditions and mental disorders are associated with higher risks for suicidal ideation or suicidal behaviors [40,51-56]. Our findings that the associations between obesity indicators (i.e., BMI, waist circumference, and waist-height ratio) and suicidal ideation diminished in the present study after controlling for either chronic conditions or current depression or both suggest that impaired physical health or mental disorders may have individually (for overall obese women) or jointly (for abdominally obese women) contributed to an increased risk for suicidal ideation among obese population.

Our study has several limitations. First, suicidal ideation was assessed based on self-report of a single item on the PHQ-9 and thus subject to recall bias. Second, although we have included a number of physical chronic conditions in our analyses, the severity of each individual chronic condition was unknown, so we were unable to weigh differently the impact of a specific chronic condition on the association between obesity indictors and suicidal ideation. Third, we 
TABLE 3: Regression coefficients $(\beta)$ with standard errors (SEs) for suicidal ideation among US women aged $\geq 20$ years by continuous BMI, waist circumference, and waist-height ratio*, NHANES 20052008.

\begin{tabular}{lccc}
\hline & $\beta$ & SE & $\begin{array}{c}P \text { for linear } \\
\text { trend }\end{array}$ \\
\hline Body mass index $\left(\mathrm{kg} / \mathrm{m}^{2}\right)$ & & & \\
Model 1 & 0.043 & 0.011 & $<0.001$ \\
Model 2 & 0.048 & 0.010 & $<0.001$ \\
Model 3 & 0.029 & 0.012 & 0.020 \\
Model 4 & 0.031 & 0.015 & 0.047 \\
Model 5 & 0.022 & 0.016 & 0.166 \\
Waist circumference $(\mathrm{cm})$ & & & \\
Model 1 & 0.023 & 0.004 & $<0.001$ \\
Model 2 & 0.024 & 0.004 & $<0.001$ \\
Model 3 & 0.015 & 0.005 & 0.010 \\
Model 4 & 0.015 & 0.006 & 0.025 \\
Model 5 & 0.011 & 0.007 & 0.117 \\
Waist-height ratio & & & \\
Model 1 & 4.419 & 0.808 & $<0.001$ \\
Model 2 & 4.128 & 0.779 & $<0.001$ \\
Model 3 & 2.630 & 0.934 & 0.008 \\
Model 4 & 2.762 & 1.076 & 0.015 \\
Model 5 & 2.102 & 1.034 & 0.073 \\
\hline
\end{tabular}

* Model 1: unadjusted; Model 2, adjusted for demographic characteristics (age, race, education, marital status, poverty-income ratio) and lifestylerelated behaviors (current smoking, excessive drinking, and physical activity); Model 3: further adjusted for the number of chronic conditions (including diabetes, hypertension, congestive heart failure, coronary artery disease, stroke, arthritis, asthma, chronic bronchitis, emphysema, liver disease, thyroid disease, renal diseases, sleep disorders, cancer, and disability); Model 4: further adjusted for current depression; Model 5: further adjusted for both the number of chronic conditions and current depression.

assessed the potential role of current depression in confounding the relationship of obesity with suicidal ideation in the present study; however, we were unable to evaluate the possible effects of other psychiatric disorders, antipsychotic medication use, emotional functioning, social support, and family history of suicidal behaviors on the association due to lack of data. Finally, potentially protective factors including life satisfaction, social support, and coping were not assessed either.

In conclusion, our results from this large, populationbased study suggest that depression and chronic physical conditions may explain much of the association between obesity and suicidal ideation among US adult women. Although the exact mechanisms mediating the association between obesity and suicidal ideation remain to be elucidated, results from the present study may have important implications for preventing suicidal ideation. Our findings suggest that combined intervention programs targeting obesity management and the prevention/treatment of obesityrelated physical chronic conditions and depression may help to reduce the prevalence of suicidal ideation and ultimately reduce risk of suicide mortality. Currently, obesity among US adults is a major public health concern after decade of increase in its prevalence $[2,57,58]$. Thus, efforts on screening and assessing obesity-related physical and mental disorders may provide useful information for preventing suicidal behaviors in this population.

\section{Conflict of Interests}

The authors declare that they have no conflict of interests.

\section{Disclaimer}

The findings and conclusions in this paper are those of the authors and do not necessarily represent the official position of the Centers for Disease Control and Prevention.

\section{References}

[1] K. M. Flegal, M. D. Carroll, C. L. Ogden, and L. R. Curtin, "Prevalence and trends in obesity among US adults, 19992008," Journal of the American Medical Association, vol. 303, no. 3, pp. 235-241, 2010.

[2] E. S. Ford, C. Li, G. Zhao, and J. Tsai, "Trends in obesity and abdominal obesity among adults in the United States from 1999-2008," International Journal of Obesity, vol. 35, no. 5, pp. 736-743, 2011.

[3] C. L. Ogden, S. Z. Yanovski, M. D. Carroll, and K. M. Flegal, "The Epidemiology of Obesity," Gastroenterology, vol. 132, no. 6, pp. 2087-2102, 2007.

[4] O. Bjerkeset, P. Romundstad, J. Evans, and D. Gunnell, "Association of adult body mass index and height with anxiety, depression, and suicide in the general population: the HUNT study," American Journal of Epidemiology, vol. 167, no. 2, pp. 193-202, 2008.

[5] J. Ma and L. Xiao, "Obesity and depression in US women: results from the 2005-2006 national health and nutritional examination survey," Obesity, vol. 18, no. 2, pp. 347-353, 2010.

[6] A. A. Mather, B. J. Cox, M. W. Enns, and J. Sareen, "Associations of obesity with psychiatric disorders and suicidal behaviors in a nationally representative sample," Journal of Psychosomatic Research, vol. 66, no. 4, pp. 277-285, 2009.

[7] G. Zhao, E. S. Ford, S. Dhingra, C. Li, T. W. Strine, and A. H. Mokdad, "Depression and anxiety among US adults: associations with body mass index," International Journal of Obesity, vol. 33, no. 2, pp. 257-266, 2009.

[8] G. Zhao, E. S. Ford, C. Li, J. Tsai, S. Dhingra, and L. S. Balluz, "Waist circumference, abdominal obesity, and depression among overweight and obese U.S. adults: national health and nutrition examination survey 2005-2006," BMC Psychiatry, vol. 11, article 130, 2011.

[9] G. D. Batty, E. Whitley, M. Kivimäki, P. Tynelius, and F. Rasmussen, "Body mass index and attempted suicide: cohort study of 1,133,019 Swedish men," American Journal of Epidemiology, vol. 172, no. 8, pp. 890-899, 2010.

[10] M. S. Kaplan, B. H. McFarland, and N. Huguet, "The relationship of body weight to suicide risk among men and women: results from the US National Health Interview Survey linked mortality file," Journal of Nervous and Mental Disease, vol. 195, no. 11, pp. 948-951, 2007.

[11] P. K. E. Magnusson, F. Rasmussen, D. A. Lawlor, P. Tynelius, and D. Gunnell, "Association of body mass index with suicide mortality: a prospective cohort study of more than one million 
men," American Journal of Epidemiology, vol. 163, no. 1, pp. 18, 2006.

[12] K. J. Mukamal, I. Kawachi, M. Miller, and E. B. Rimm, "Body mass index and risk of suicide among men," Archives of Internal Medicine, vol. 167, no. 5, pp. 468-475, 2007.

[13] K. J. Mukamal, E. B. Rimm, I. Kawachi, E. J. O’Reilly, E. E. Calle, and M. Miller, "Body mass index and risk of suicide among one million US adults," Epidemiology, vol. 21, no. 1, pp. 82-86, 2010.

[14] C. Diaz-Sastre, E. Baca-Garcia, M. M. Perez-Rodriguez et al., "Low plasma cholesterol levels in suicidal males: a genderand body mass index-matched case-control study of suicide attempters and nonattempters," Progress in Neuro-Psychopharmacology and Biological Psychiatry, vol. 31, no. 4, pp. 901-905, 2007.

[15] J. D. Fernstrom and R. J. Wurtman, "Brain serotonin content: physiological dependence on plasma tryptophan levels," Science, vol. 173, no. 3992, pp. 149-152, 1971.

[16] B. A. Golomb, L. Tenkanen, T. Alikoski et al., "Insulin sensitivity markers - predictors of accidents and suicides in Helsinki Heart Study screenees," Journal of Clinical Epidemiology, vol. 55, no. 8, pp. 767-773, 2002.

[17] D. Lipsett, B. K. Madras, R. J. Wurtman, and H. N. Munro, "Serum tryptophan level after carbohydrate ingestion: selective decline in non-albumin-bound tryptophan coincident with reduction in serum free fatty acids," Life Sciences, vol. 12, no. 2, pp. 57-64, 1973.

[18] K. M. Carpenter, D. S. Hasin, D. B. Allison, and M. S. Faith, "Relationships between obesity and DSM-IV major depressive disorder, suicide ideation, and suicide attempts: results from a general population study," American Journal of Public Health, vol. 90, no. 2, pp. 251-257, 2000.

[19] Centers for Disease Control and Prevention. National Center for Health Statistics: National Health and Nutrition Examination Survey, 2012, http://www.cdc.gov/nchs/nhanes/nhanes _questionnaires.htm/.

[20] A. Martin, W. Rief, A. Klaiberg, and E. Braehler, "Validity of the Brief Patient Health Questionnaire Mood Scale (PHQ-9) in the general population," General Hospital Psychiatry, vol. 28, no. 1, pp. 71-77, 2006.

[21] K. Kroenke, R. L. Spitzer, and J. B. W. Williams, "The PHQ-9: validity of a brief depression severity measure," Journal of General Internal Medicine, vol. 16, no. 9, pp. 606-613, 2001.

[22] N. Lossnitzer, T. Müller-Tasch, B. Löwe et al., "Exploring potential associations of suicidal ideation and ideas of selfharm in patients with congestive heart failure.," Depression and anxiety, vol. 26, no. 8, pp. 764-768, 2009.

[23] J. A. Sirey, M. L. Bruce, M. Carpenter et al., "Depressive symptoms and suicidal ideation among older adults receiving home delivered meals," International Journal of Geriatric Psychiatry, vol. 23, no. 12, pp. 1306-1311, 2008.

[24] M. G. Tektonidou, A. Dasgupta, and M. M. Ward, "Suicidal ideation among adults with arthritis: prevalence and subgroups at highest risk. Data from the 2007-2008 National Health and Nutrition Examination Survey," Arthritis Care \& Research, vol. 63, no. 9, pp. 1322-1333, 2011.

[25] J. Walker, C. H. Hansen, L. Hodges et al., "Screening for suicidality in cancer patients using Item 9 of the nine-item patient health questionnaire; does the item score predict who requires further assessment?" General Hospital Psychiatry, vol. 32, no. 2, pp. 218-220, 2010.

[26] J. Walker, C. H. Hansen, I. Butcher et al., "Thoughts of death and suicide reported by cancer patients who endorsed the "suicidal thoughts" item of the PHQ-9 during routine screening for depression," Psychosomatics, vol. 52, no. 5, pp. 424-427, 2011.

[27] A. V. Chobanian, G. L. Bakris, H. R. Black et al., "Seventh report of the Joint National Committee on Prevention, Detection, Evaluation, and Treatment of High Blood Pressure," Hypertension, vol. 42, no. 6, pp. 1206-1252, 2003.

[28] K. Kroenke, T. W. Strine, R. L. Spitzer, J. B. W. Williams, J. T. Berry, and A. H. Mokdad, "The PHQ-8 as a measure of current depression in the general population," Journal of Affective Disorders, vol. 114, no. 1-3, pp. 163-173, 2009.

[29] T. W. Strine, A. H. Mokdad, L. S. Balluz et al., "Depression and anxiety in the United States: findings from the 2006 Behavioral Risk Factor Surveillance system," Psychiatric Services, vol. 59, no. 12, pp. 1383-1390, 2008.

[30] A. Must, J. Spadano, E. H. Coakley, A. E. Field, G. Colditz, and W. H. Dietz, "The disease burden associated with overweight and obesity," Journal of the American Medical Association, vol. 282, no. 16, pp. 1523-1529, 1999.

[31] S. Paeratakul, J. C. Lovejoy, D. H. Ryan, and G. A. Bray, “The relation of gender, race and socioeconomic status to obesity and obesity comorbidities in a sample of US adults," International Journal of Obesity, vol. 26, no. 9, pp. 1205-1210, 2002.

[32] S. G. Bruce, N. D. Riediger, J. M. Zacharias, and T. K. Young, "Obesity and obesity-related comorbidities in a Canadian First Nation population," Preventing chronic disease, vol. 8, no. 1, p. A03, 2011.

[33] G. Gariepy, J. Wang, A. Lesage, and N. Schmitz, "Obesity and the risk of disability in a 12-year cohort study: the role of psychological distress," Social Psychiatry and Psychiatric Epidemiology, pp. 1-7, 2010.

[34] G. Gariepy, D. Nitka, and N. Schmitz, "The association between obesity and anxiety disorders in the population: a systematic review and meta-analysis," International Journal of Obesity, vol. 34, no. 3, pp. 407-419, 2010.

[35] D. P. Guh, W. Zhang, N. Bansback, Z. Amarsi, C. L. Birmingham, and A. H. Anis, "The incidence of co-morbidities related to obesity and overweight: a systematic review and metaanalysis," BMC Public Health, vol. 9, article no. 88, 2009.

[36] S. Kasen, P. Cohen, H. Chen, and A. Must, "Obesity and psychopathology in women: a three decade prospective study," International Journal of Obesity, vol. 32, no. 3, pp. 558-566, 2008.

[37] F. S. Luppino, L. M. De Wit, P. F. Bouvy et al., "Overweight, obesity, and depression: a systematic review and meta-analysis of longitudinal studies," Archives of General Psychiatry, vol. 67, no. 3, pp. 220-229, 2010.

[38] J. Gensichen, A. Teising, J. König, F. M. Gerlach, and J. J. Petersen, "Predictors of suicidal ideation in depressive primary care patients," Journal of Affective Disorders, vol. 125, no. 1-3, pp. 124-127, 2010.

[39] M. K. Nock, G. Borges, E. J. Bromet, C. B. Cha, R. C. Kessler, and S. Lee, "Suicide and suicidal behavior," Epidemiologic Reviews, vol. 30, no. 1, pp. 133-154, 2008.

[40] M. K. Nock, G. Borges, E. J. Bromet et al., "Cross-national prevalence and risk factors for suicidal ideation, plans and attempts," British Journal of Psychiatry, vol. 192, no. 2, pp. 98$105,2008$.

[41] P. R. Casey, G. Dunn, B. D. Kelly et al., "Factors associated with suicidal ideation in the general population: five-centre analysis from the ODIN study," British Journal of Psychiatry, vol. 189, pp. 410-415, 2006.

[42] A. M. Miniño, J. Q. Xu, and K. D. Kochanek, "Deaths: preliminary data for 2008," National Vital Statistics Reports, vol. 59 no. 2, National Center for Health Statistics, Hyattsville, Md, 
USA, 2010, http://www.cdc.gov/nchs/data/nvsr/nvsr59/nvsr59 -02.pdf.

[43] M. Nordentoft, "Prevention of suicide and attempted suicide in Denmark. Epidemiological studies of suicide and intervention studies in selected risk groups," Danish Medical Journal, vol. 54, no. 4, pp. 306-369, 2007.

[44] R. C. Kessler, P. Berglund, G. Borges, M. Nock, and P. S. Wang, "Trends in suicide ideation, plans, gestures, and attempts in the United States, 1990-1992 to 2001-2003," Journal of the American Medical Association, vol. 293, no. 20, pp. 2487-2495, 2005.

[45] A. E. Crosby, B. Han, L. A. Ortega, S. E. Parks, and J. Gfroerer, "Suicidal thoughts and behaviors among adults aged $>/=18$ years-United States, 2008-2009," Morbidity and Mortality Weekly Report, vol. 60, no. 13, pp. 1-22, 2011.

[46] H. C. Kung, J. L. Pearson, and X. Liu, "Risk factors for male and female suicide decedents ages 15-64 in the United Statesresults from the 1993 National Mortality Followback Survey," Social Psychiatry and Psychiatric Epidemiology, vol. 38, no. 8, pp. 419-426, 2003.

[47] M. D. Llorente, M. Burke, G. R. Gregory et al., "Prostate cancer: a significant risk factor for late-life suicide," American Journal of Geriatric Psychiatry, vol. 13, no. 3, pp. 195-201, 2005.

[48] H. T. Robertson and D. B. Allison, "Drugs associated with more suicidal ideations are also associated with more suicide attempts," PLoS ONE, vol. 4, no. 10, Article ID e7312, 2009.

[49] M. E. J. Lean, T. S. Han, and J. C. Seidell, "Impairment of health and quality of life in people with large waist circumference," The Lancet, vol. 351, no. 9106, pp. 853-856, 1998.

[50] A. C. Rivenes, S. B. Harvey, and A. Mykletun, "The relationship between abdominal fat, obesity, and common mental disorders: results from the HUNT Study," Journal of Psychosomatic Research, vol. 66, no. 4, pp. 269-275, 2009.

[51] J. M. Bolton, J. Pagura, M. W. Enns, B. Grant, and J. Sareen, “A population-based longitudinal study of risk factors for suicide attempts in major depressive disorder," Journal of Psychiatric Research, vol. 44, no. 13, pp. 817-826, 2010.

[52] S. J. Garlow, J. Rosenberg, J. D. Moore et al., "Depression, desperation, and suicidal ideation in college students: results from the American Foundation for Suicide Prevention College Screening Project at Emory University," Depression and Anxiety, vol. 25, no. 6, pp. 482-488, 2008.

[53] J. MacLean, D. J. Kinley, F. Jacobi, J. M. Bolton, and J. Sareen, "The relationship between physical conditions and suicidal behavior among those with mood disorders," Journal of Affective Disorders, vol. 130, no. 1-2, pp. 245-250, 2011.

[54] M. K. Nock, I. Hwang, N. A. Sampson, and R. C. Kessler, "Mental disorders, comorbidity and suicidal behavior: results from the national comorbidity survey replication," Molecular Psychiatry, vol. 15, no. 8, pp. 868-876, 2010.

[55] J. Sareen, B. J. Cox, T. O. Afifi et al., "Anxiety disorders and risk for suicidal ideation and suicide attempts: a population-based longitudinal study of adults," Archives of General Psychiatry, vol. 62, no. 11, pp. 1249-1257, 2005.

[56] K. M. Scott, I. Hwang, W. T. Chiu et al., "Chronic physical conditions and their association with first onset of suicidal behavior in the world mental health surveys," Psychosomatic Medicine, vol. 72, no. 7, pp. 712-719, 2010.

[57] M. A. Beydoun and Y. Wang, "Gender-ethnic disparity in BMI and waist circumference distribution shifts in US adults," Obesity, vol. 17, no. 1, pp. 169-176, 2009.
[58] C. Li, E. S. Ford, L. C. McGuire, and A. H. Mokdad, "Increasing trends in waist circumference and abdominal obesity among U.S. adults," Obesity, vol. 15, no. 1, pp. 216-224, 2007. 


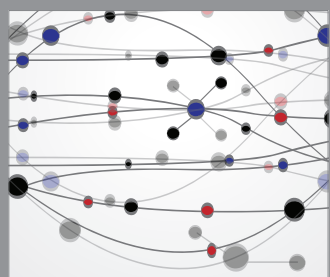

The Scientific World Journal
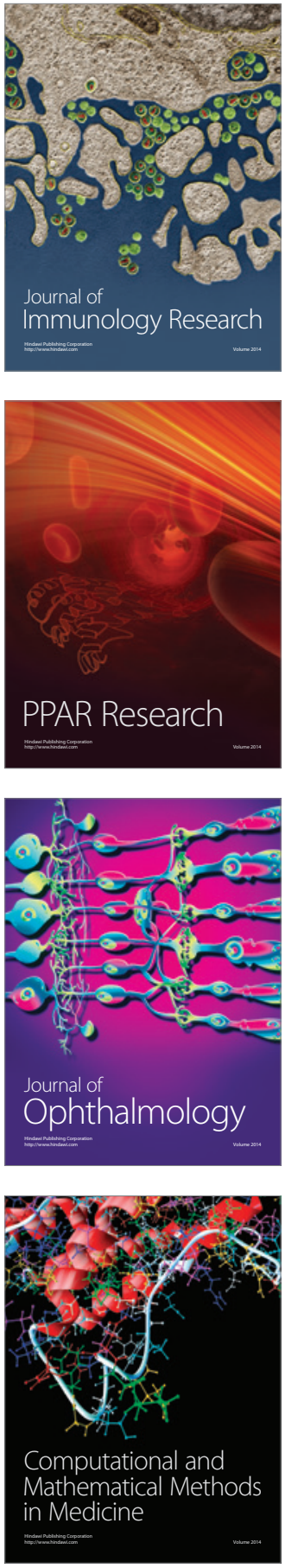

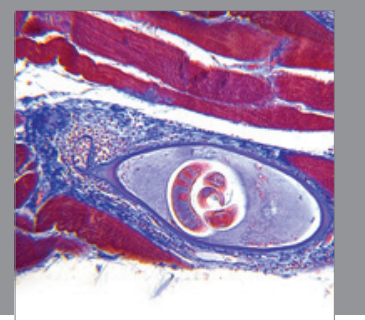

Gastroenterology

Research and Practice
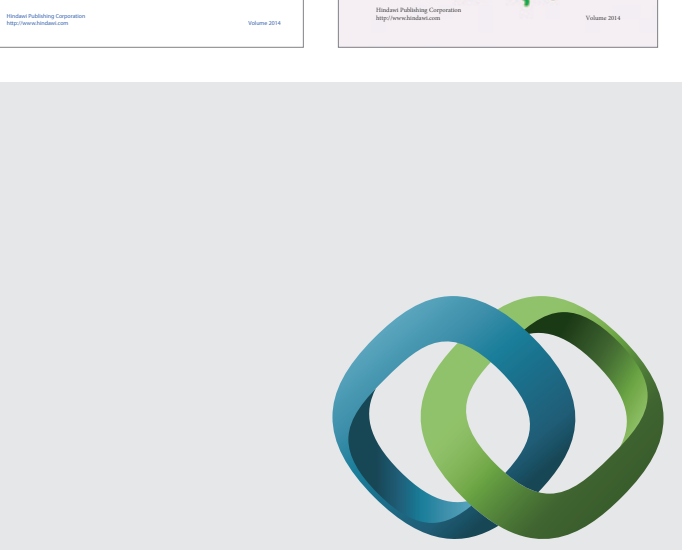

\section{Hindawi}

Submit your manuscripts at

http://www.hindawi.com
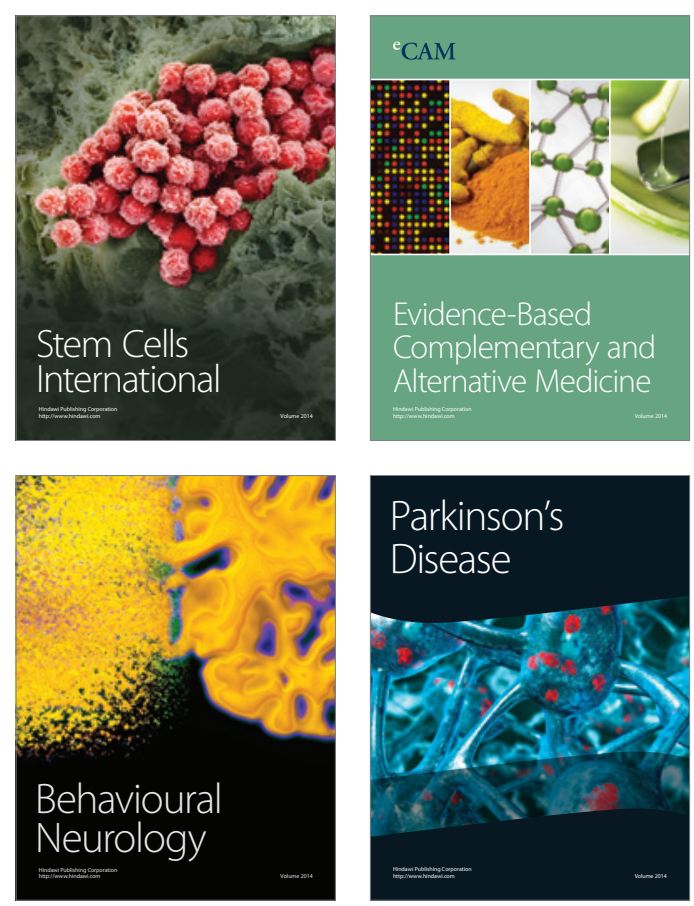

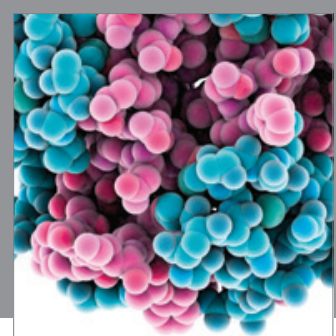

Journal of
Diabetes Research

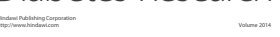

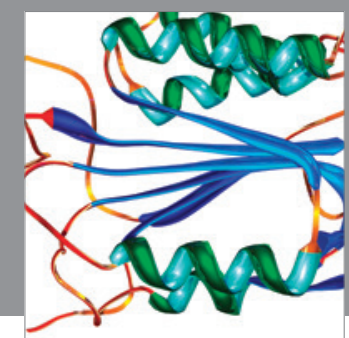

Disease Markers
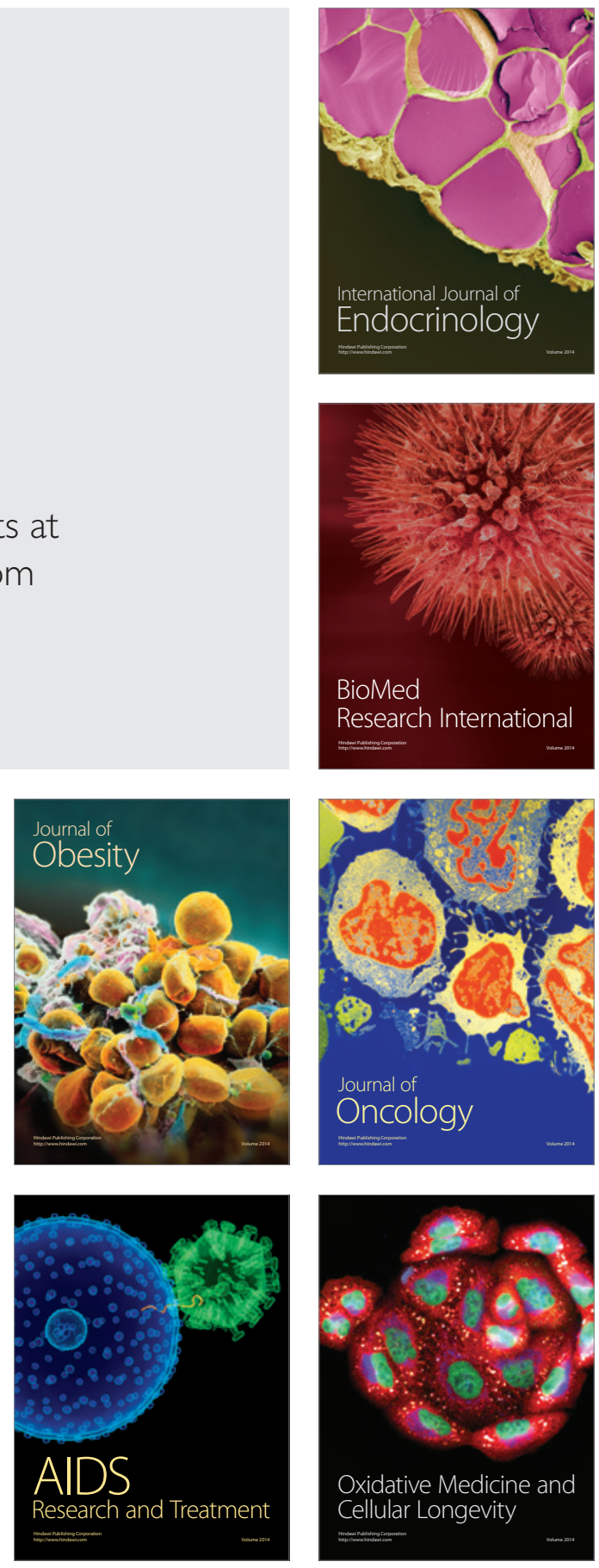